\title{
Two-Stage Elephant Trunk approach for open management of distal aortic arch and descending aortic pathology in patients with Marfan syndrome
}

\author{
Camilo A. Velasquez ${ }^{1}$, Mohammad A. Zafar ${ }^{1}$, Ayman Saeyeldin ${ }^{1}$, Syed Usman Bin Mahmood ${ }^{1}$, Adam J. \\ Brownstein $^{1}$, Young Erben ${ }^{2}$, Bulat A. Ziganshin ${ }^{1,3}$, John A. Elefteriades ${ }^{1}$ \\ ${ }^{1}$ Aortic Institute at Yale-New Haven Hospital, Yale University School of Medicine, New Haven, CT, USA; ${ }^{2}$ Section of Vascular and Endovascular \\ Surgery, Yale-New Haven Hospital, CT, USA; ${ }^{3}$ Department of Surgical Diseases \#2, Kazan State Medical University, Kazan, Russia \\ Correspondence to: John A. Elefteriades, MD. Aortic Institute at Yale-New Haven, Yale University School of Medicine, Clinic Building CB 317,789 \\ Howard Avenue, New Haven, CT 06519, USA. Email: john.elefteriades@yale.edu.
}

Submitted Nov 16, 2017. Accepted for publication Nov 17, 2017.

doi: $10.21037 /$ acs.2017.11.11

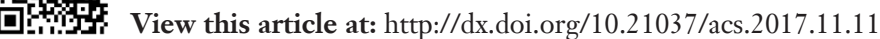

\section{Introduction}

Open surgical treatment of an aortic pathology (aneurysm or dissection) afflicting the entire length of the thoracic aorta is a surgical challenge (1). Therefore, a two-stage procedure for "mega-aorta" syndrome was instituted to address this surgical dilemma (2). However, Borst and colleagues observed that with the classical technique (conventional arch replacement followed by descending aortic replacement), mobilization of the previous graft at the distal arch anastomosis was cumbersome and could result in injury to the pulmonary artery, the aorta itself, the esophagus, and other surrounding structures (3). Therefore, in 1992 they introduced the Elephant Trunk technique, which was later modified by Crawford and Svensson (4).

Connective tissue disorders such as Marfan syndrome increase the complexity of management of aortic arch and descending aortic pathology. This autosomal dominant disease is characterized by the presence of cardiovascular disorders affecting mostly the thoracic aorta with an associated decrease in life expectancy (5). The weak tissues and characteristic distal progression of aneurysmal disease in Marfan syndrome have led to the recommendation for aggressive early surgical intervention in the ascending aorta and preemptive aortic arch replacement when a Type A dissection is the presenting event $(6,7)$. The two-stage Elephant Trunk technique fits into this aggressive paradigm and facilitates the correction of coexistent or subsequent descending aortic dilatation (5). In this paper, we describe the technical essentials of both stages of the Elephant Trunk procedure as practiced extensively at our institution.

\section{The Elephant Trunk procedure: operative} technique

The two stages of the Elephant Trunk technique are employed when surgery of both the ascending and descending aorta is required (Figure 1). Usually, Stage 1 is separated from Stage 2 by an interval of 3 to 6 weeks (8) so as to allow healing of the body and recovery of cardiopulmonary function. However, if the patient is symptomatic and the risk of rupture is high, the interval must be shortened (1).

\section{Stage 1 Elephant Trunk: aortic arch/ascending aorta intervention}

Surgical technique

\section{Exposure and cannulation}

Stage 1 is performed through a standard median sternotomy with oblique extension into the left neck (if needed) for appropriate exposure of the arch vessels.

Axillary cannulation is the preferred inflow for cardiopulmonary bypass due to the usual presence of thrombus, calcification, and mobile atherosclerotic plaques in the dilated descending aorta, rendering femoral cannulation and retrograde perfusion extraordinarily 

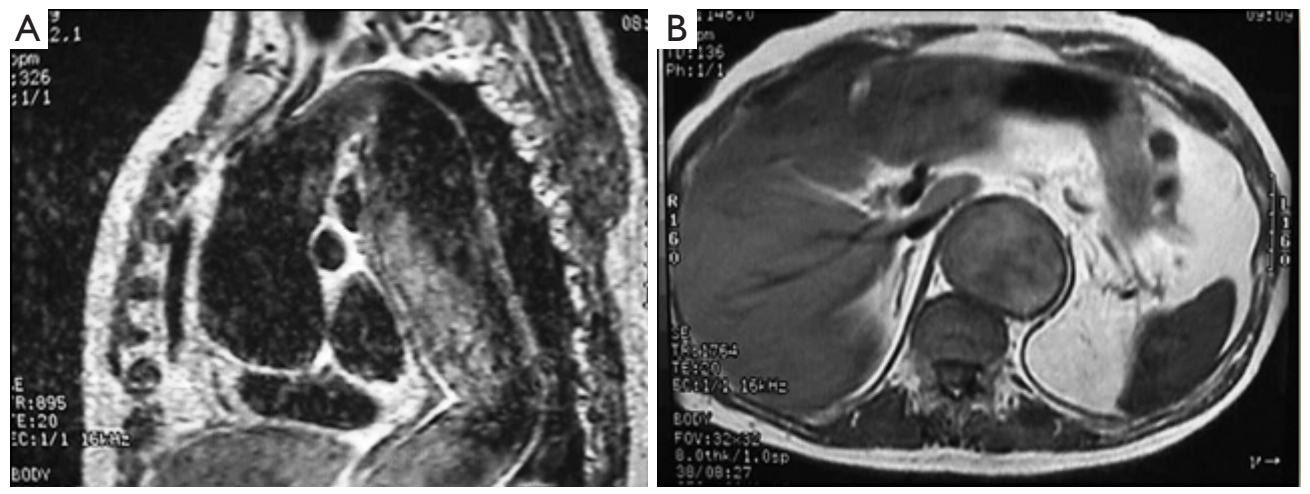

Figure 1 Magnetic resonance imaging showing dilation of the thoracic aorta in both, the ascending and the descending portions. (A) Sagittal view; (B) axial view.

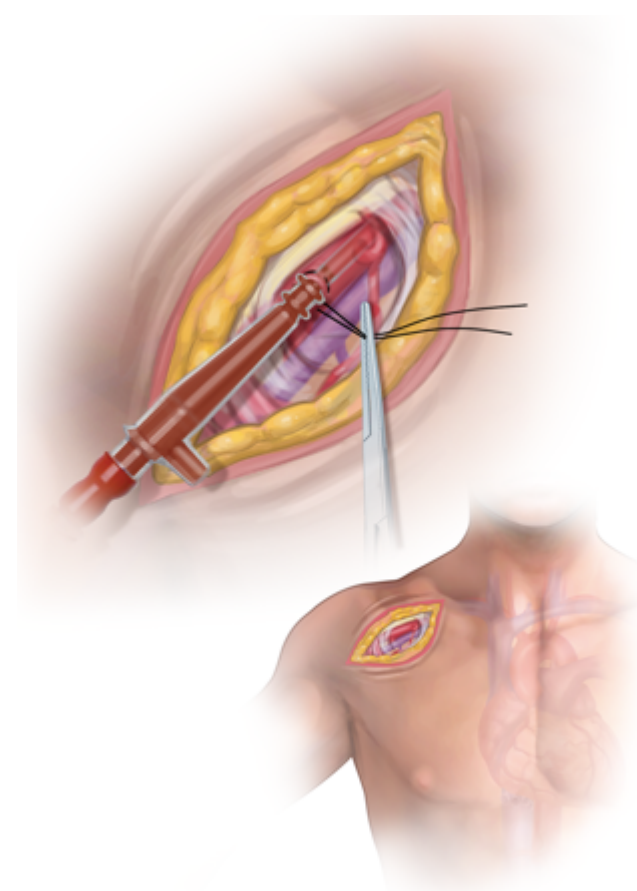

Figure 2 Axillary cannulation (Seldinger technique).

dangerous. A $5-8 \mathrm{~cm}$ incision is made in the right subclavicular region and the axillary artery exposed and controlled. The brachial plexus is meticulously protected (Figure 2). We currently cannulate the axillary artery through a pursestring by Seldinger technique [in a similar fashion as we have described previously for femoral cannulation (9)] (Figure 3). A two-staged venous cannula is placed via the right atrium and a left heart vent is positioned in the right superior pulmonary vein.

Very often, but not routinely, the left innominate vein is mobilized, controlled and divided for better exposure of the arch vessels which are then mobilized during the preliminary dissections of the mediastinum (Figure 4). Exposure of the arch and wide, safe spreading of the sternotomy are greatly enhanced by innominate vein division, which is uniformly well tolerated without permanent sequelae in our experience (10).

\section{Cardiopulmonary bypass}

Standard cardiopulmonary bypass is instituted. Straight deep hypothermic arrest (DHCA) is utilized during the arch replacement and elephant trunk placement. We do not use perfusion techniques, either antegrade or retrograde. We cool to $18{ }^{\circ} \mathrm{C}$. The head is packed in ice. We warm to $34{ }^{\circ} \mathrm{C}$ (keeping the temperature gradient between water bath and venous return no greater than $10{ }^{\circ} \mathrm{C}$ ). We do not use transcranial oximetry or electroencephalographic monitoring. The details and outcomes of our DCHA techniques have been published previously (11).

\section{Main procedure}

After full exposure of the arch vessels is achieved, the distal ascending aorta is cross-clamped and the first dose of cardioplegia is given. During the cooling phase, the aortic root, ascending aorta and/or aortic valve are replaced if indicated (Figure 5). When the nasopharyngeal temperature reaches $18-20{ }^{\circ} \mathrm{C}$, the patient is put in the Trendelenburg position, circulatory arrest is instituted and the cross-clamp is removed.

The head vessels are mobilized as a Carrel patch, or are individually transected at their junctions with the aortic arch (Figure 6). We may include all three vessels (innominate, left carotid, left subclavian) in the repair, or we may confine the resection and re-anastomosis to the 


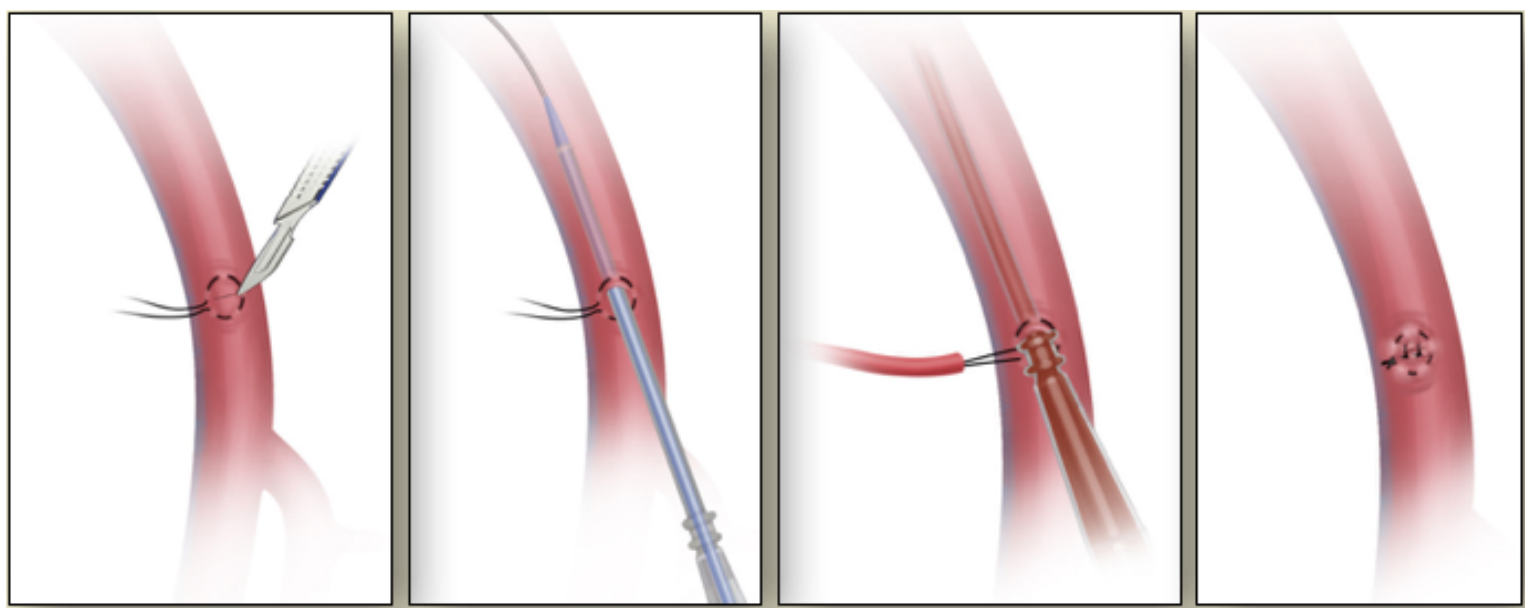

Figure 3 Seldinger technique for cannulation.
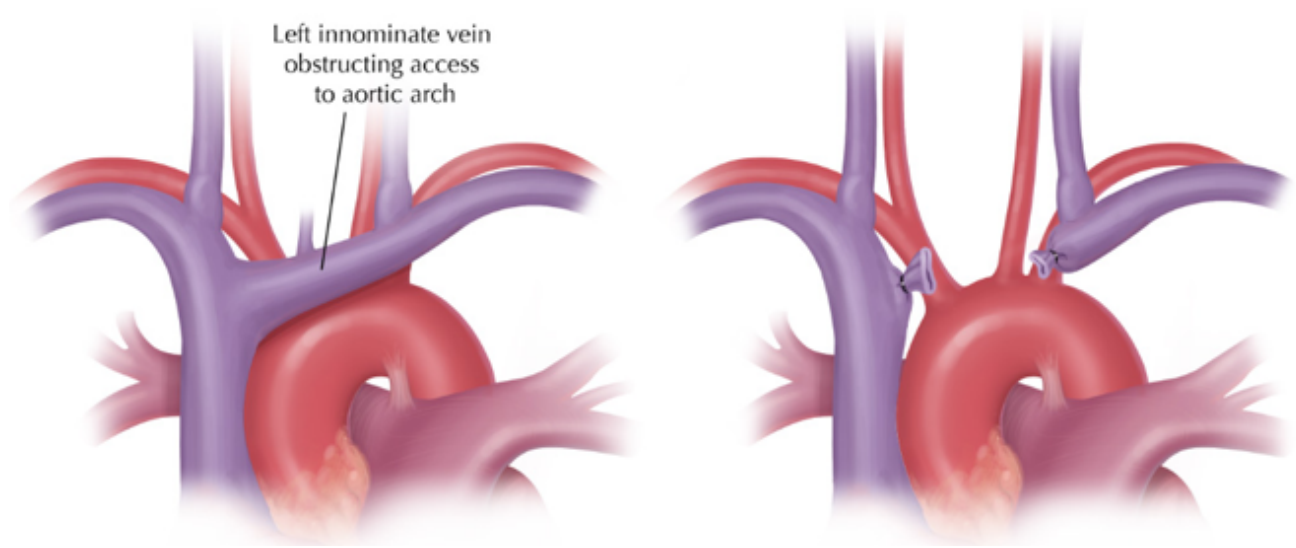

Figure 4 Innominate vein division.

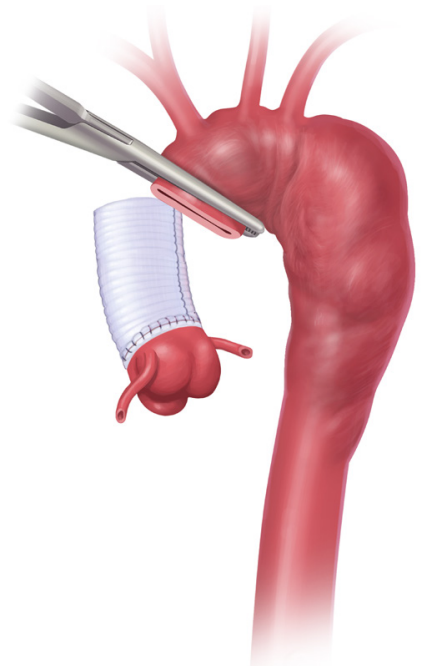

innominate and left carotid. This two-vessel approach is becoming more popular with us and worldwide. This permits the anastomosis to be performed between the left carotid and left subclavian arteries. At this level, exposure is excellent and anastomosis is facile; also, in case of bleeding, the bleeding site can usually be exposed directly for control. When the two-vessel approach is taken, the left subclavian artery is resected and bypassed at the time of the Stage II procedure. We sometimes use a branched graft, but invaginating the elephant trunk with all the bulky branches can be a bit cumbersome. The two-vessel approach also brings the anastomosis far proximal to the vagus, recurrent laryngeal, and phrenic nerves, which are thus protected.

A Dacron graft is brought to the surgical field and is invaginated into itself (Figure 7). It is important to undersize

Figure 5 Proximal aortic work during the cooling phase. the graft compared to the size of the aorta on CT scan, for 


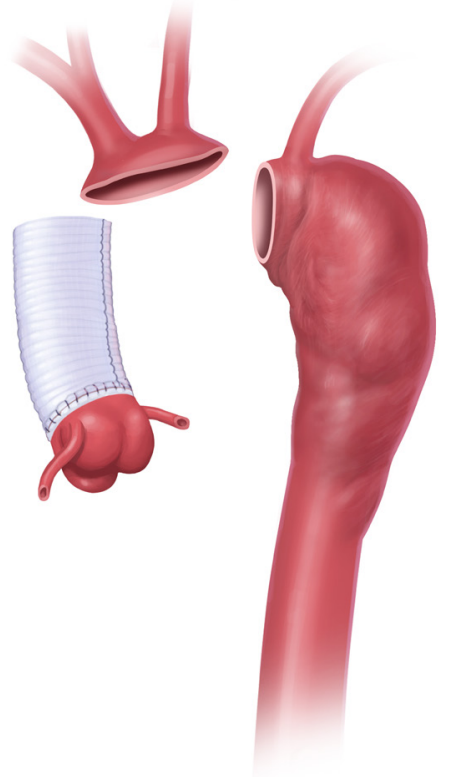

Figure 6 Mobilization of the head vessels as a carrel patch.

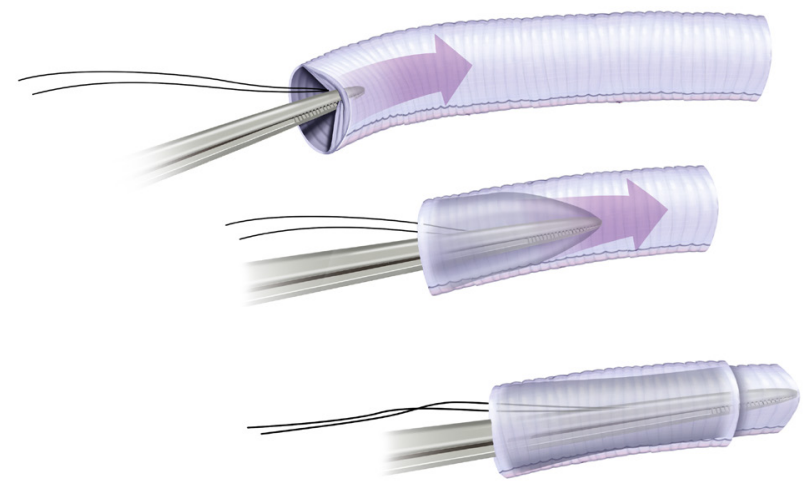

Figure 7 Elephant trunk manufacture.

several reasons. The graft must be able to be passed into the descending aorta without abrasion of the wall or kinking of the graft. It is desirable for the graft to be a bit smaller than the aorta to permit retrograde perfusion of the subclavian artery around the elephant trunk, in case of a two-vessel anastomosis. Direct visualization of the descending aorta is performed for evaluation of dissection flaps, contained rupture, and mobile atheromas, followed by graft insertion. An anastomosis is performed between the aortic tissue and the full thickness of the invaginated graft (two layers) with running suture (Figure 8). We reinforce the exterior of the aortic wall with a strip of Teflon felt. We pass a tonsil sucker into the lumen to make certain that there is no

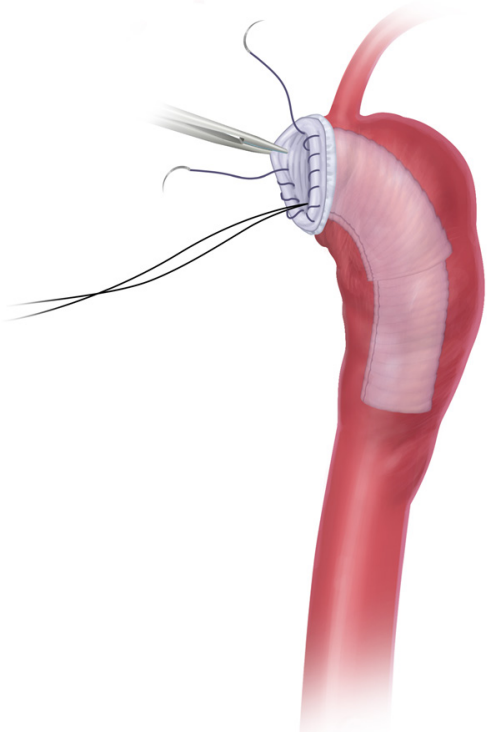

Figure 8 Elephant trunk implantation and suturing.

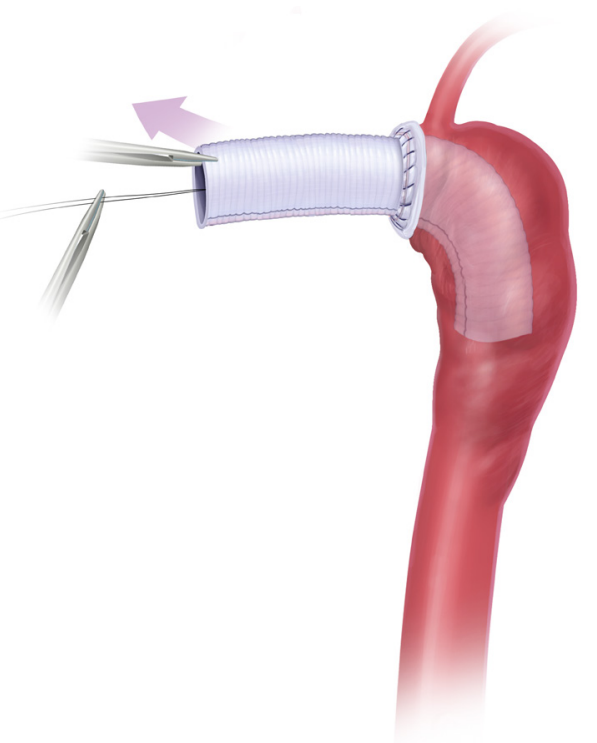

Figure 9 Elephant trunk evagination.

kink or other obstruction; palpation via the sucker is quite informative.

The elephant trunk graft inner portion is now evaginated, leaving the elephant trunk itself dangling in the descending aorta. Forceps and a nerve hook can aid in evagination of the elephant trunk (Figure 9). Arch vessel reconstruction is performed with an end-to-side anastomosis of the Carrel patch to the top of the main graft 


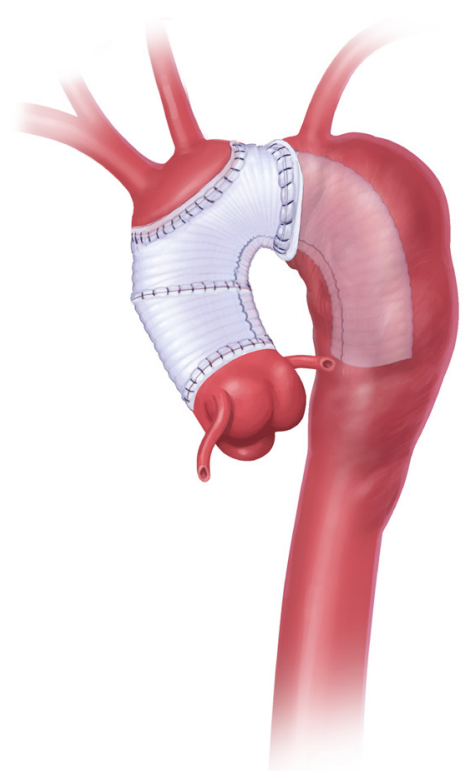

Figure 10 Head vessels and graft to graft anastomosis.

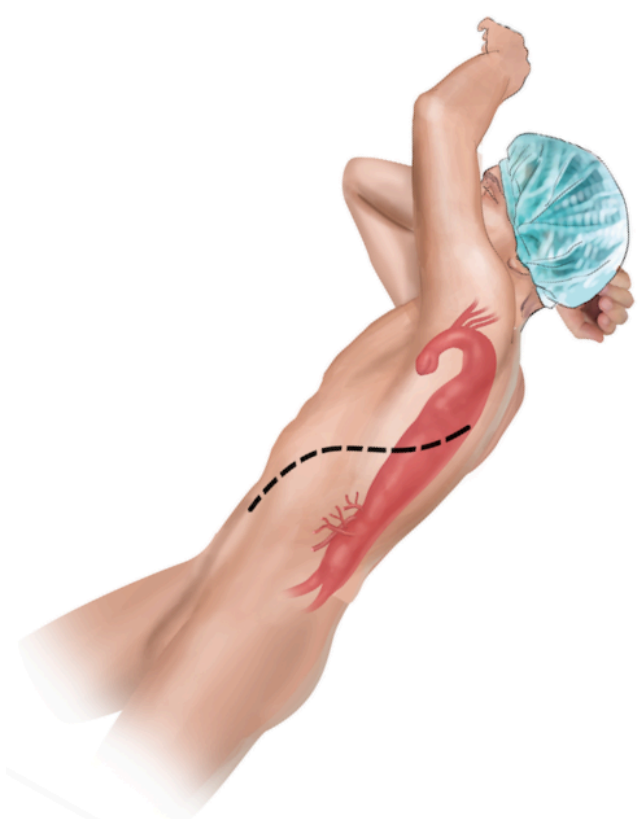

Figure 11 Patient positioning for the second stage Elephant trunk procedure.

(Figure 10). Alternatively, the side arms of a branched graft are sewn end-to-end to the transected great vessels. Another option is to use interposition grafts sewn end-to-side to the main aortic graft and end-to-end to each transected arch vessel. For this purpose, we use a 10 or $12 \mathrm{~mm}$ Dacron graft for the innominate artery and an $8 \mathrm{~mm}$ Dacron graft for the left carotid and for the left subclavian.

After completion of the aortic arch anastomoses, the aorta is de-aired meticulously and a cross-clamp is applied obliquely below the great vessel anastomoses, permitting resumption of flow to the body, with the heart still excluded. At this point, DHCA is terminated, pump flow is increased gradually, and rewarming begun. Ice is removed from the head. The air-based warmer for the body is activated.

During rewarming, the proximal graft to aortic graft anastomosis is performed (usually angled, like a "pipe fitting”), and any remaining proximal aortic work completed as needed (aortic valve, ascending aorta, aortic root or coronary buttons) (Figure 10).

Rewarming usually requires approximately 1 hour for a standard sized male patient. Once the bladder temperature reaches $34^{\circ} \mathrm{C}$, the patient is weaned from cardiopulmonary bypass.

\section{Stage 2 Elephant Trunk: descending thoracic aorta/thoracoabdominal aorta}

\section{Surgical technique}

\section{Preparation}

Most patients recover well and quickly from Stage I, as median sternotomy is generally well tolerated. In preparation for the procedure, CT angiography or magnetic resonance angiography for localization of the spinal artery are performed. We recommend that a CT scan of the aorta be done between Stage I and Stage II to visualize the arch repair and the elephant trunk.

General anesthesia is induced with a double-lumen endotracheal tube, cerebral spinal fluid drainage is used to optimize cord protection, and motor evoked potential monitoring is used to provide information about spinal cord function and anterior spinal artery perfusion.

\section{Exposure and cannulation}

After general anesthesia is induced, the patient is positioned in the right lateral decubitus with the shoulders placed at 90 degrees and the hips externally rotated to 45 degrees from horizontal (Figure 11). A left thoracotomy or thoracoabdominal incision is made (space varies depending on the segments that need to be resected) in a curvilinear fashion extending below the level of the umbilicus for complete thoracoabdominal replacement. If an iliac aneurysm must be repaired, the incision is extended toward the pubis. 
The diaphragm is incised circumferentially to protect the phrenic nerve and to preserve $2-3 \mathrm{~cm}$ of diaphragmatic tissue peripherally for later closure. We find that dividing the diaphragm circumferentially with a GIA stapler provides reinforced, clear-cut edges for later approximation. A retroperitoneal approach is used for exposure of the abdominal aorta with medial visceral rotation of the peritoneal structures for better exposure. Cannulation of the left superior or inferior pulmonary vein and femoral artery are performed for institution of left atrial to femoral bypass, which optimizes organ protection and reduces the cardiac strain with improvement in the ability of the patient to tolerate the aortic clamping.

\section{Main procedure}

Localization of the elephant trunk graft in the descending

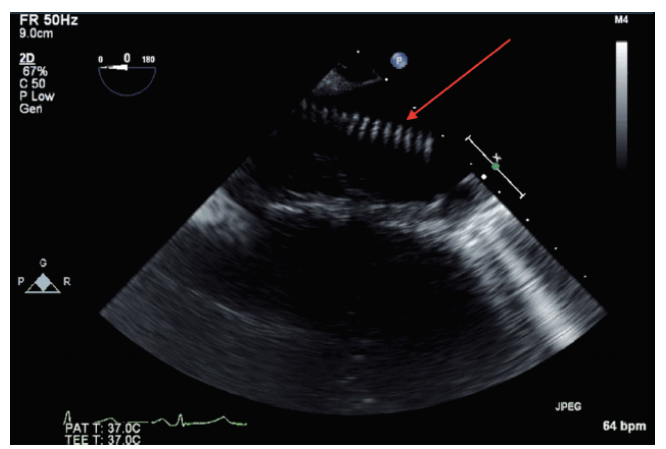

Figure 12 Transesophageal echocardiographic identification of the elephant trunk in the descending aorta.

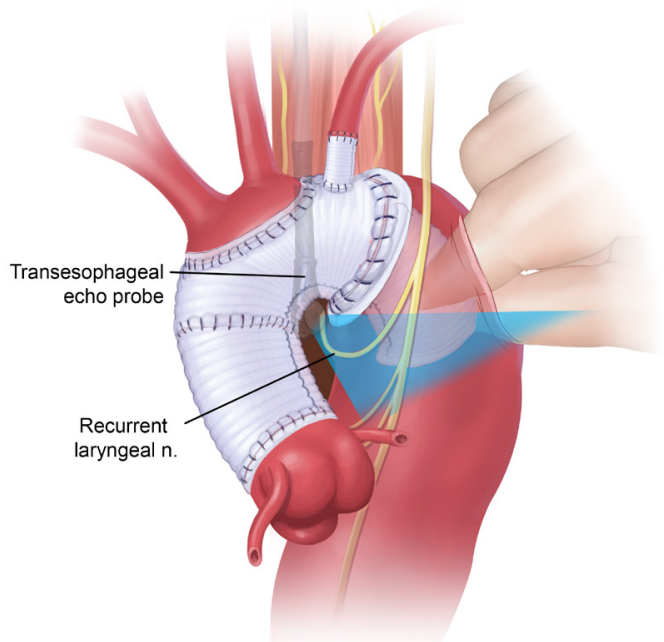

aorta is made by transesophageal echocardiography and epi-aortic ultrasound (Figure 12). After the distal aorta is clamped, left atrial to femoral bypass is instituted, adenosine is administered for cardiac standstill $(16 \mathrm{mg})$ and a vertical aortotomy is done in the descending aorta at the level of the elephant trunk graft, which is then retrieved by the fingerthumb technique. After digital retrieval of the graft, the elephant trunk is clamped (Figure 13). We like to quickly place an initial clamp on the elephant trunk for hemostasis (the patient can quickly exsanguinate). However, the graft is often folded longitudinally on itself. With the initial clamp in place, we readjust and place a new clamp squarely, without any folding of the graft, above the original clamp, which is then removed. It is now important to pull down gently on the elephant trunk, as a considerable longitudinal portion of the elephant trunk graft may still be up in the aortic arch. Without "pulling down" the free upper portion of the elephant trunk, the eventual completed graft, after distal anastomosis, will be too long and thus susceptible to kinking.

We have published a textual and video description of alternative techniques for elephant trunk retrieval (12).

The distal anastomosis is now performed (often with an interposition tube graft if the elephant trunk is not long enough to reach without augmentation). We often perform a beveled distal anastomosis to permit inclusion of critical posterior intercostal arteries. We use external Teflon felt on the aorta. We reinforce the posterior portion of the anastomosis with interrupted mattress sutures before the main anterior portion of the anastomosis is completed.

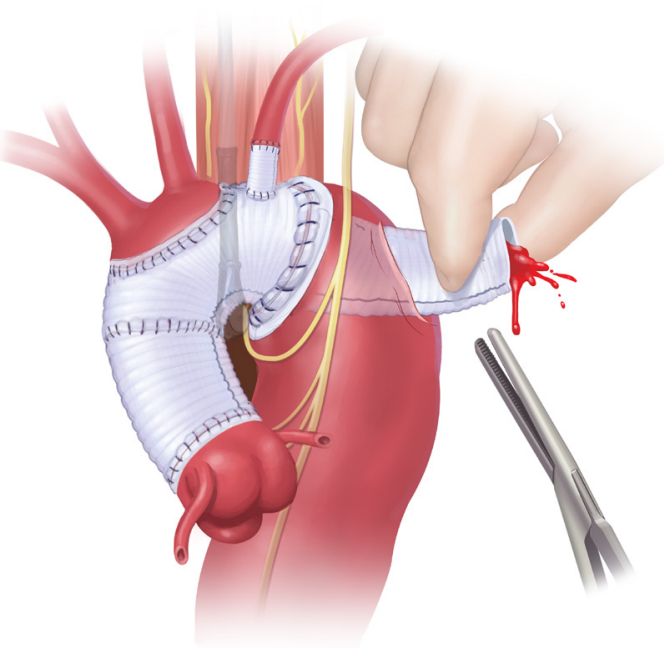

Figure 13 Finger-thumb technique for retrieval of the elephant trunk. 
A

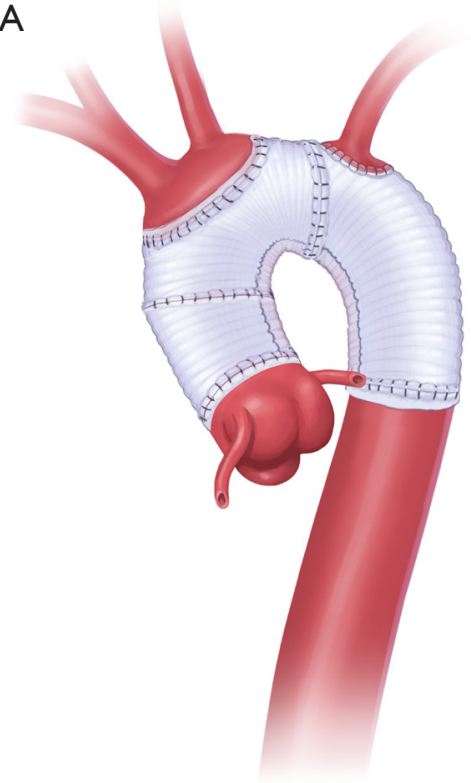

B

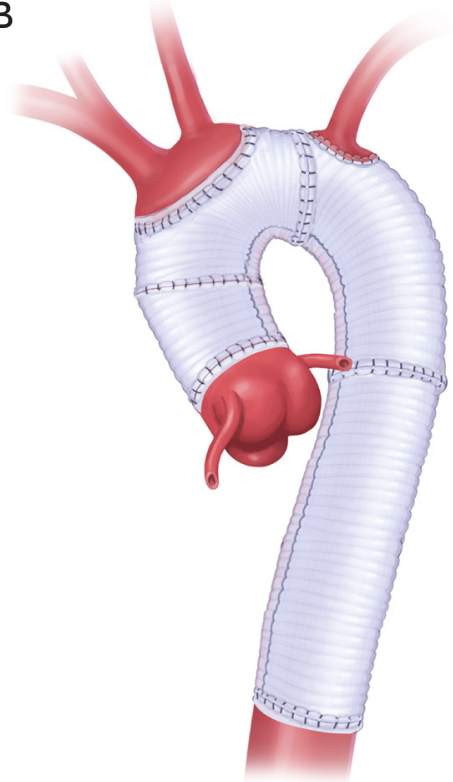

Figure 14 Completion of the Elephant Trunk procedure. (A) Anastomosis to the descending aorta; (B) Replacement of the thoracoabdominal aorta.

The anastomosis may be done in the thorax or at any level in the abdomen, even including the entirety of the thoracoabdominal aorta, as dictated by the specific anatomy (Figure 14).

Left heart bypass is terminated and the aorta is allowed to fill. Biological (e.g., Evicel) or chemical glue (e.g., BioGlue) may be used for reinforcement of the suture lines. We have published on the safety of BioGlue for aortic anastomoses (without predisposing to false aneurysm formation) (13). The remaining aortic tissue is wrapped around the anastomosed graft and the thoracotomy/thoraco-abdominal incision is closed in a standard fashion.

\section{Comments}

The thoracic aorta is commonly involved progressively in Marfan syndrome through the natural course of the disease. A "progressive" involvement of the aortic segments is common, with aortic root aneurysm being the hallmark initial site, and enlargement of the remaining ascending aorta, aortic arch, descending, and thoracoabdominal aorta ensuing and prompting additional surgical procedures (5).

Multiple studies have addressed the advisability of preemptive arch replacement when an aortic root aneurysm or Type A dissection is encountered. Schoenhoff et al. (6) concluded that Marfan patients have a small risk of re- interventions on the aortic arch after elective aortic root replacement. However, if the surgical procedure is performed after a Type A dissection, the risk of reintervention increases ( $3 \%$ vs. 33\%; $\mathrm{P}=0.0001)$. Furthermore, Bachet et al. (7) found similar results, concluding that aortic arch replacement is not indicated during elective aortic root surgery. Nonetheless, over decades, the significant likelihood of aneurysmal dilatation in the remnant aortic arch after surgery for Type A aortic dissection is an incentive for considering an aggressive approach towards the aortic arch. Thus, a staged procedure that involves the placement of an elephant trunk during Stage I facilitates the operative approach in anticipated Stage II.

The application of the staged Elephant Trunk procedure is thought to decrease the pulmonary complications and comorbidities associated with techniques that require full exposure of the proximal and distal segments of the thoracic aorta, such as the clamshell approach pioneered by Kouchoukos $(14,15)$. Dr. Kouchoukos has achieved superb results with a one-stage clamshell procedure, but these have not been widely replicated. Also, by pre-supplying a free-flowing elephant trunk graft, the proximal descending aorta does not need to be dissected, thereby avoiding injury to the adjacent structures such as the pulmonary artery, esophagus, and recurrent laryngeal nerve. Furthermore, after the aorta is incised, the elephant trunk can be easily 
secured and clamped under direct visualization, thus decreasing the critical clamp time during the descending procedure. Most importantly, the Elephant Trunk procedure avoids the need for DHCA during Stage II. As fond as we are of DHCA for Stage I, we generally dislike DHCA for descending aortic procedures. We reserve its use to the unclampable proximal descending aorta in cases of large descending and thoracoabdominal aortic aneurysms. We do not like to perfuse from below across the diseased thoracoabdominal aorta to the brain. We dread losing particles in the left decubitus position into the proximal aortic root.

Several additional points merit attention. In patients unfit for an open Stage II, either intrinsically or because of impaired recovery after Stage I, a non-invasive stent graft approach provides a valuable alternative (16). The use of the frozen elephant trunk procedure during Stage I and thoracic endovascular aortic repair in patients with connective tissue disease is controversial due to the fragility of the tissue and the risk of ongoing dilatation in the native aortic tissue after stenting. Another stent related uncertainty has to do with fear of non-durability, as many Marfan patients experience their first aortic procedure at a young age. Many experts recommended stenting in Marfan disease only as a temporary bridge to definitive open repair $(5,16)$.

For cerebral protection, we have in this chapter recommended straight DHCA, the most commonly used technique at our institution. We have shown this to be safe and effective when instituted for a duration of 50 minutes or less, with an overall mortality of $2.4 \%$ and a $1.6 \%$ rate of stroke $(11,17)$. However, antegrade perfusion techniques are facile in the present era of axillary cannulation; we have no qualms whatsoever with application of antegrade perfusion in the Marfan setting discussed in this paper. However, we recommend careful attention to choices regarding vessels perfused and perfusion rate and pressure (18).

Complete resection of the arch vessels with graft replacement is recommended in Marfan patients to avoid late aneurysmal dilatation of the remaining tissue and consequent need for re-intervention.

An appropriate length of the elephant trunk must be placed $(10-15 \mathrm{~cm})$ in order to avoid paraplegia via thrombus formation and embolic events (2).

Also, reinforcement of the suture lines is recommended when intervening in patients with connective tissue disorders.

Finally, the prophylactic placement of an elephant trunk graft in Marfan patients with a normal or mildly dilated descending aorta is advised when procedures involving the aortic arch are performed. This provides a secure proximal landing zone for later replacement of the descending thoracic aorta, which may become aneurysmal and increase the future risk of life-threatening aortic events if left unattended (5).

The brilliant Elephant Trunk technique conceived by the great pioneer Dr. Borst continues to serve Marfan and other patients with the most extensive aortic disease very well.

\section{Acknowledgements}

None.

\section{Footnote}

Conflicts of Interest: The authors have no conflicts of interest to declare.

\section{References}

1. Svensson LG. The elephant trunk procedure: uses in complex aortic diseases. Curr Opin Cardiol 2005;20:491-5.

2. Tanaka A, Estrera AL. Elephant Trunk: Argument for All Arches. Semin Cardiothorac Vasc Anesth 2016;20:322-6.

3. Borst HG. The birth of the elephant trunk technique. J Thorac Cardiovasc Surg 2013;145:44.

4. Crawford ES, Coselli JS, Svensson LG, et al. Diffuse aneurysmal disease (chronic aortic dissection, Marfan, and mega aorta syndromes) and multiple aneurysm. Treatment by subtotal and total aortic replacement emphasizing the elephant trunk operation. Ann Surg 1990;211:521-37.

5. Miyahara S, Okita Y. Overview of current surgical strategies for aortic disease in patients with Marfan syndrome. Surg Today 2016;46:1006-18.

6. Schoenhoff FS, Kadner A, Czerny M, et al. Should aortic arch replacement be performed during initial surgery for aortic root aneurysm in patients with Marfan syndrome? Eur J Cardiothorac Surg 2013;44:346-51; discussion 51.

7. Bachet J, Larrazet F, Goudot B, et al. When should the aortic arch be replaced in Marfan patients? Ann Thorac Surg 2007;83:S774-9; discussion S85-90.

8. Coselli JS, Trocciola SM, de la Cruz KI, et al. Arch Aneurysms. In: Kaiser LR, Kron IL, Spra TL. editors. Mastery of Cardiothoracic Surgery. Third edition. Philadelphia, USA: Lippincott Williams and Wilkins, 2015:599-610.

9. Tsiouris A, Elkinany S, Ziganshin BA, et al. Open Seldinger-Guided Femoral Artery Cannulation 
Technique for Thoracic Aortic Surgery. Ann Thorac Surg 2016;101:2231-5.

10. Sai Sudhakar CB, Elefteriades JA. Safety of left innominate vein division during aortic arch surgery. Ann Thorac Surg 2000;70:856-8.

11. Ziganshin BA, Rajbanshi BG, Tranquilli M, et al. Straight deep hypothermic circulatory arrest for cerebral protection during aortic arch surgery: Safe and effective. J Thorac Cardiovasc Surg 2014;148:888-98; discussion 98-900.

12. Ziganshin BA, Elefteriades JA. Finger-Thumb Technique for Elephant Trunk Retrieval. Aorta (Stamford) 2013;1:286-8.

13. Elefteriades JA. "How I do it: utilization of high-pressure sealants in aortic reconstruction". J Cardiothorac Surg 2009; 4:27.

14. Kouchoukos NT, Masetti P, Rokkas CK, et al. Singlestage reoperative repair of chronic type A aortic dissection by means of the arch-first technique. J Thorac Cardiovasc

Cite this article as: Velasquez CA, Zafar MA, Saeyeldin A, Bin Mahmood SU, Brownstein AJ, Erben Y, Ziganshin BA, Elefteriades JA. Two-Stage Elephant Trunk approach for open management of distal aortic arch and descending aortic pathology in patients with Marfan syndrome. Ann Cardiothorac Surg 2017;6(6):712-720. doi: 10.21037/acs.2017.11.11
Surg 2001;122:578-82.

15. Shimizu H, Takahashi T, Yamazaki M, et al. Extensive total arch replacement via clamshell incision in a patient with aortic arch aneurysm and Stanford type B aortic dissection. Gen Thorac Cardiovasc Surg 2008;56:183-6.

16. Sun L, Li M, Zhu J, et al. Surgery for patients with Marfan syndrome with type A dissection involving the aortic arch using total arch replacement combined with stented elephant trunk implantation: the acute versus the chronic. J Thorac Cardiovasc Surg 2011;142:e85-91.

17. Dumfarth J, Ziganshin BA, Tranquilli M, et al. Cerebral protection in aortic arch surgery: hypothermia alone suffices. Tex Heart Inst J 2013;40:564-5.

18. Elefteriades JA, Ziganshin BA. Brain protection in aortic arch surgery: antegrade cerebral perfusion and retrograde cerebral perfusion need a tougher row to hoe. J Thorac Cardiovasc Surg 2014;148:2902-4. 